\title{
Amino acids in liver disease
}

By LaRs Hagenfeldt, Department of Clinical Chemistry, Karolinska Hospital, Stockholm, Sweden and LjuSk Siw ERIKsson and John Wahren, Departments of Medicine and Clinical Physiology, Huddinge Hospital, Stockholm, Sweden

The steady-state concentrations of amino acids seen in the basal post-absorptive state in man reflect the net balance between release from endogenous protein stores, primarily muscle, and utilization by various tissues, primarily the liver. After an overnight fast there is a net release of amino acids from skeletal muscle, the output of alanine and glutamine exceeding that of all other amino acids and accounting for more than $50 \%$ of the total amino nitrogen released (Figs. I and 2). Corresponding to the negative amino acid balance of muscle, there is a consistent uptake of amino acids across the splanchnic vascular bed. Again, alanine and glutamine predominate in the amino acid uptake. Within the splanchnic area the liver is the site of alanine uptake while glutamine is being taken up primarily by the gut and subsequently released in part as alanine to the liver. The major site of glutamine disposal is the kidney, where it provides $\mathbf{N}$ for ammoniagenesis.

The above outline for the major amino acid fluxes in the basal state does not involve all individual amino acids. Thus, glutamate is being released by the splanchnic tissues and taken up by skeletal muscle, while serine is utilized by muscle as well as by splanchnic tissues (Fig. I). The branched-chain amino acids

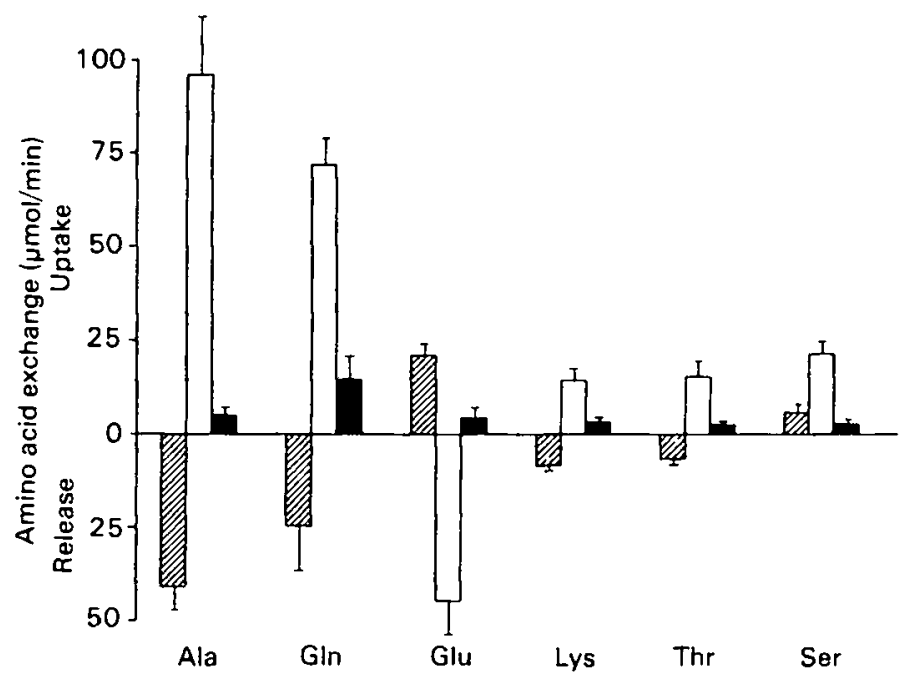

Fig. I. Net exchange of amino acids in the splanchnic area ( $\square$ ), leg muscle ( legs) and the brain ( $\square$ ) in healthy subjects in the post-absorptive state. Data from Hagenfeldt et al. (1980) and Sato et al. (1981). Brain uptake was calculated assuming an average cerebral blood flow of $0.8 \mathrm{l} / \mathrm{min}$. Standard errors of the means are represented by vertical bars. 


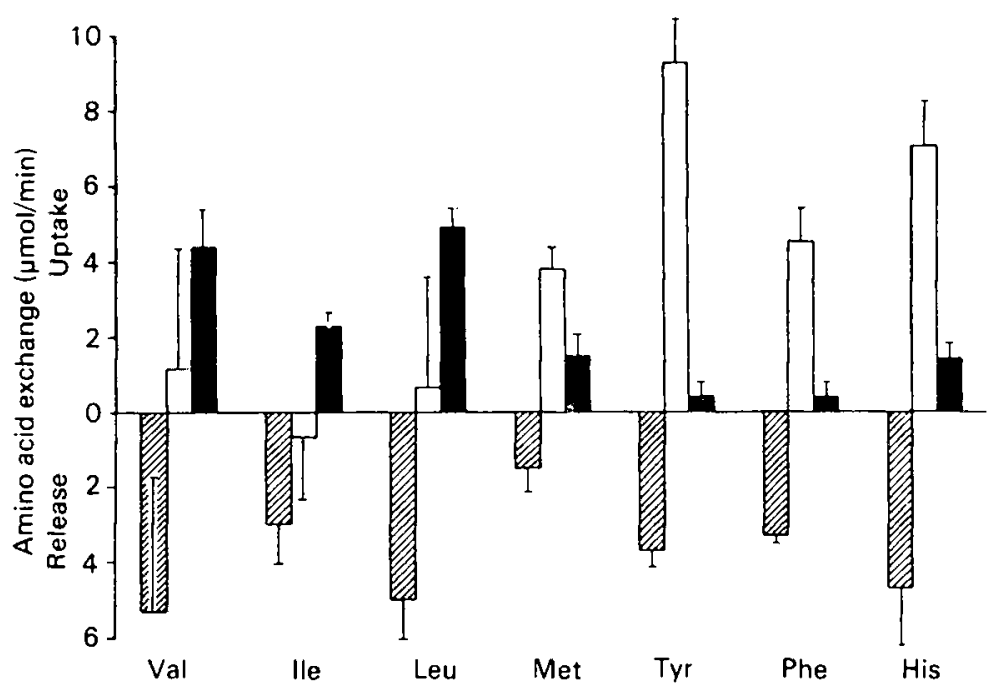

Fig. 2. As in Fig. 1 . Note the difference in magnitude of scaling.

(BCAA) have attracted particular interest in recent years in view of their possible roles in the regulation of muscle protein turnover as well as in the pathogenesis of hepatic encephalopathy (for a review, see Eriksson \& Wahren, 1982). For the BCAA, there is a small release from muscle in the basal state, no measurable net exchange across the splanchnic bed and utilization by the brain to match the muscle output (Fig. 2).

It is the purpose of this brief presentation to review the inter-relationships of amino acid concentrations in the basal state in man. In addition, the possible pathogenic and therapeutic roles of the BCAA in hepatic encephalopathycurrently a subject of much speculation-will be discussed and the result of some recent controlled clinical trials presented.

\section{Plasma amino acid concentrations and membrane transport systems}

Amino acids are lipid insoluble and their passage across biological membranes depend on carrier-mediated transport. Several such transport systems with varying affinities for different amino acids have been described (Christensen, 1975). A study of the distribution of amino acids between plasma and erythrocytes (Hagenfeldt \& Arvidsson, I980) has shown that the ratios of erythrocyte:plasma concentrations $(E: P)$ are influenced by at least two of these transport systems. In addition, the plasma levels themselves appear to be determined to a large extent by the activity of transport systems. This can be illustrated by using the statistical technique of factor analysis applied to the plasma amino acid concentrations of a group of healthy subjects (Table I). This statistical method is used to reduce the dimensionality of the covariance structure of a set of variables, in this case the plasma concentrations of individual amino acids (Chatfield \& Collins, I980). New variables, called factors, are derived. The factor loadings (presented in Table I) are measures of the covariance of each amino acid concentration with the factor and 
Table I. Varimax rotated factor matrix for amino acids in plasma in fifty healthy men

\begin{tabular}{llrlrrrrrr} 
Factor & Amino acid & \multicolumn{1}{c}{ I } & II & \multicolumn{1}{c}{ III } & \multicolumn{1}{c}{ IV } & \multicolumn{1}{c}{ V } & \multicolumn{1}{c}{ VI } & \multicolumn{1}{c}{ VII } & \multicolumn{1}{l}{$h^{2}$} \\
I & Isoleucine & 0.89 & -0.01 & -0.06 & 0.08 & 0.05 & 0.23 & -0.09 & 0.86 \\
& Leucine & 0.85 & 0.03 & -0.02 & 0.31 & 0.10 & 0.21 & 0.27 & 0.95 \\
& Valine & 0.84 & 0.04 & -0.10 & 0.19 & 0.23 & 0.03 & -0.08 & 0.82 \\
& Phenylalanine & 0.60 & 0.27 & 0.19 & 0.10 & 0.05 & 0.46 & 0.45 & 0.89 \\
& Tyrosine & 0.54 & 0.45 & -0.11 & 0.20 & 0.17 & 0.02 & 0.27 & 0.65 \\
& Tryptophan & 0.43 & 0.39 & -0.08 & 0.23 & 0.11 & 0.40 & 0.03 & 0.57 \\
& Histidine & 0.37 & 0.35 & 0.20 & 0.33 & 0.07 & 0.26 & 0.11 & 0.49 \\
II & Alanine & 0.06 & 0.90 & 0.21 & 0.14 & 0.07 & 0.24 & -0.14 & 0.95 \\
& Arginine & -0.07 & 0.40 & 0.13 & 0.38 & 0.27 & 0.08 & 0.01 & 0.41 \\
III & Serine & -0.13 & -0.07 & 0.82 & 0.10 & 0.24 & 0.17 & 0.07 & 0.80 \\
& Ornithine & 0.24 & 0.20 & 0.64 & -0.14 & -0.58 & -0.13 & -0.08 & 0.89 \\
& Glycine & -0.16 & 0.09 & 0.55 & -0.07 & 0.07 & 0.24 & -0.14 & 0.37 \\
& Threonine & 0.12 & 0.33 & 0.47 & 0.10 & 0.07 & 0.00 & 0.14 & 0.38 \\
IV & Cystine & 0.26 & 0.09 & -0.02 & 0.84 & -0.08 & 0.21 & 0.07 & 0.84 \\
& a-Aminobutyrate & 0.47 & 0.14 & -0.07 & 0.61 & -0.12 & -0.12 & -0.22 & 0.70 \\
& Lysine & 0.31 & 0.35 & 0.26 & 0.42 & 0.01 & 0.01 & 0.01 & 0.46 \\
V & Glutamine & 0.34 & 0.06 & 0.13 & 0.04 & 0.58 & 0.00 & -0.02 & 0.48 \\
& Proline & 0.16 & 0.12 & 0.13 & -0.01 & 0.53 & -0.10 & -0.04 & 0.35 \\
& Asparagine & 0.01 & 0.31 & 0.09 & -0.12 & 0.49 & 0.32 & 0.03 & 0.46 \\
& Glutamate & 0.18 & 0.13 & -0.11 & 0.33 & -0.47 & -0.20 & 0.35 & 0.55 \\
VI & Methionine & 0.23 & 0.13 & 0.24 & 0.09 & 0.09 & 0.76 & 0.00 & 0.72 \\
& Taurine & 0.34 & 0.12 & 0.02 & 0.04 & -0.10 & 0.38 & -0.13 & 0.31 \\
VII & Aspartate & -0.02 & -0.06 & 0.09 & -0.04 & -0.07 & -0.03 & 0.85 & 0.75
\end{tabular}

Factors I-VII are measures of the covariance of each amino acid concentration with the factor (see this page).

$h^{2}$ is the proportion of the total variance of each amino acid that can be explained by the factor structure.

the communality $\left(h^{2}\right)$ is the proportion of the total variance of each amino acid that can be explained by the factor structure. The following 'biological explanation' to the factor structure (Table I) may be offered.

Factor $I$ includes all those amino acids, except methionine, that have a high affinity to the L-transport system for neutral amino acids.

Factor II is primarily related to alanine and arginine. Alanine is one of the major amino acids in the amino $\mathrm{N}$ transport from muscle to liver. The mutual correlations of alanine and arginine to factor II may reflect an association between proteolysis and urea cycle activity.

Factor III. Three of these amino acids-serine, glycine and threonine-share a common transport system, the $A$ system. The association of ornithine with this factor may be caused by the precursor-product relationship between glycine and ornithine through the action of glycine amidinotransferase ( $E C$ 2.I.4.I). 
Factor IV involves cystine and lysine, both of which are transported by the system for dibasic amino acids. Arginine, which also shares this transport system, has a high loading to factor IV.

Factor $V$ includes four amino acids-glutamine, proline, asparagine and glutamate--which are closely related metabolically. The negative factor loading for glutamate agrees with the inverse correlation observed between the $E: P$ values of glutamate and glutamine (Hagenfeldt \& Arvidsson, r980).

Factor VI. Methionine is the only amino acid with a high affinity to the L-transport system that is not included in factor I. Instead, this amino acid is related to factor VI together with taurine, one of its metabolic end-products.

It is evident from this analysis of the covariance structure of plasma amino acid levels that transport phenomena play an important role in determining these levels. It should also be noted that the communalities of several amino acids are very high. For eleven of the amino acids more than $70 \%$ of the total variance could be explained by the factor structure. Thus, exogenous factors, such as diet, could only exert a limited influence on the plasma levels of these amino acids.

\section{Plasma amino acids in patients with liver cirrhosis}

Liver failure is associated with characteristic changes in the plasma amino acids (Wu et al. 1955; Iber et al. 1957; Zinneman et al. 1969). Thus, the concentrations of the three BCAA are decreased, while tyrosine, phenylalanine and methionine are increased. Determinations of amino acid concentrations in whole blood have confirmed these results and also demonstrated a marked reduction in the level of aspartic acid (Sato et al. $198 \mathrm{r}$; Eriksson, Hagenfeldt et al. 1982). The low levels of BCAA could in part be caused by the hyperinsulinaemia seen in these patients (Collins \& Crofford, I 969), this in turn being caused by the portal-systemic shunting and/or decreased hepatic catabolism of insulin (Johnston \& Alberti, 1977; Smith-Laing et al. 1979). However, although some studies have reported an inverse relationship between the arterial levels of insulin and leucine in patients with liver cirrhosis (Sato et al. I981), others have been unable to find such a correlation (Smith-Laing et al. 1979; Iwasaki et al. 1980). This indicates that other factors, such as the hyperglucagonaemia (Marchesini et al. 1979) and raised catecholamine levels (Eriksson, Hagenfeldt et al. 1982) observed in these patients may also be involved. Finally, increased brain uptake could contribute to the low BCAA levels. The brain is a major site of removal of BCAA from plasma (Fig. 2) and the capacity for neutral amino acid transport across the blood-brain barrier is augmented in liver failure (James et al. 1978; Sato et al. 1981).

The rise in aromatic amino acids and methionine is mainly due to impaired metabolism by and shunting past the cirrhotic liver (Miller, 1962). The presence of a catabolic state with hyperglucagonaemia and increased gluconeogenesis could also contribute to the abnormal amino acid pattern (Soeters \& Fischer, 1976). Furthermore, the turnover rate and endogenous breakdown of protein has been shown to be elevated in patients with cirrhosis (O'Keefe et al. 1981). The reason for the diminished level of aspartate is so far unknown. In contrast to the finding in 
whole blood, the plasma level of this amino acid is increased (Morgan et al. 1982). This indicates that a derangement of transport could be responsible also for the changes in aspartate. Intracellular aspartate levels are reported to be forty- to fifty-fold greater than the plasma concentrations (Hagenfeldt \& Arvidsson, 1980).

\section{Plasma amino acid imbalance and hepatic encephalopathy}

The BCAA, tyrosine, phenylalanine and methionine are all transported by the $\mathrm{L}$ system (Oldendorf \& Szabo, 1976). The capacity for transport across the bloodbrain barrier is limited and, even at physiological concentrations, these amino acids compete with each other for entry into the brain (Pardridge \& Oldendorf, 1977). The increased blood levels of the aromatic amino acids together with the decreased transport competition from the BCAA may lead to an augmented uptake of aromatic amino acids and methionine in the brain. An increased blood-brain barrier permeability for neutral amino acids demonstrated in portacaval shunted rats could also contribute to the increased uptake (James et al. 1978).

The aromatic amino acids are central neurotransmitter precursors (Guroff \& Udenfriend, 1962). The increased availability of these amino acids could therefore result in an imbalance of the synthesis of serotonin, noradrenaline and dopamine in the brain (Munro et al. 1975; Fischer \& Baldessarini, 1976; James et al. 1979). This may in turn result in increased formation of 'false' neurotransmitters such as octopamine and phenylethanolamine, which are stored in the presynaptic vesicles and could function as weak neurotransmitters (Fischer \& Baldessarini, 1971). Together, these alterations have been suggested to be responsible for the cerebral dysfunction associated with hepatic encephalopathy.

In addition, the low aspartate levels may be of importance for the pathogenesis of hepatic encephalopathy. First, aspartate participates in the urea cycle and an intracellular depletion could be a limiting factor in the process of ammonia detoxification and might explain the reduced urea synthesis in liver cirrhosis (Rudman et al. 1973). Second, aspartate has been suggested as an excitatory neurotransmitter in the brain (Krnjevic, 1974). Low intracerebral levels could therefore result in neurotransmitter imbalance.

\section{Experimental studies in man}

The above considerations have led to the suggestion that BCAA administration to patients with liver coma may exert a beneficial effect on brain function (Fischer et al. 1974; Fischer \& Baldessarini, 1976) by normalizing the plasma amino acid concentration and reducing the brain influx of aromatic amino acids. This theory prompted us to investigate the metabolic effects of BCAA in healthy volunteers and in patients with liver cirrhosis. Intravenous infusion of leucine alone, resulting in a sixfold rise in its arterial concentration, was accompanied by a pronounced decrease in the blood concentrations of methionine, tyrosine and phenylalanine in healthy subjects (Hagenfeldt et al. I980). In contrast, valine infusion resulted in only a slight decrease in tyrosine concentration and isoleucine administration did not at all affect the amino acid concentrations. Thus, leucine was far more effective 


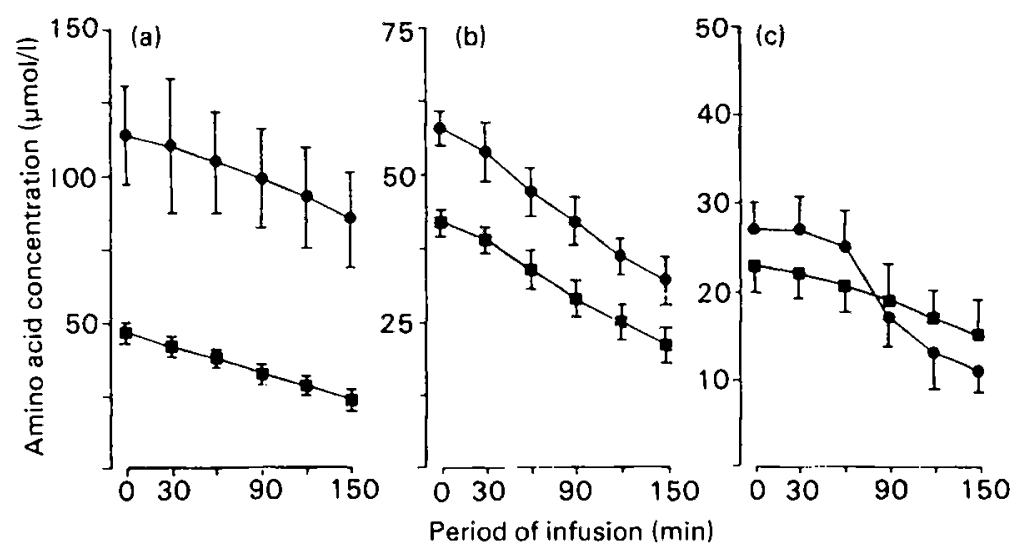

Fig. 3. Effect of intravenous infusion of a mixture of branched-chain amino acids on the blood levels of (a) tyrosine, (b) phenylalanine and (c) methionine in patients with liver cirrhosis ( ) and healthy controls $(\square)$. Standard errors of the means are represented by vertical bars.

than valine or isoleucine in influencing the aromatic amino acids (Eriksson et al. r981). However, during leucine administration a lowering was seen not only for the aromatic amino acids and methionine but also for valine and isoleucine, the other two BCAA which are already low in patients with liver cirrhosis. A mixture of all three $\mathrm{BCAA}$ ( $70 \%$ leucine, $20 \%$ valine, $10 \%$ isoleucine) was therefore tried and this solution was shown to effectively decrease the blood levels of tyrosine, phenylalanine and methionine in both healthy subjects and in patients with liver cirrhosis (Fig. 3) (Sato et al. 1981).

The brain exchange of amino acids was studied in the basal state and during leucine infusion in healthy subjects (Hagenfeldt et al. 1980). Catheters were introduced percutaneously into an artery and the right-sided jugular vein and the brain's uptake of amino acids was estimated from the arterial-jugular venous (A-V) concentration differences. During leucine infusion the brain's uptake of methionine and isoleucine was inhibited. No effect could be demonstrated on the aromatic amino acids since no net exchange of these across the brain could be observed in the basal state.

A similar study was performed using the mixed BCAA solution in patients with liver cirrhosis and in healthy control subjects (Sato et al. 1981). In the basal state, uptake by the brain was shown for all three BCAA, glutamine, serine and lysine in both groups, while an uptake of tyrosine and phenylalanine was found in the patients only. It is noteworthy that the uptake of the BCAA was similar in both groups; the lower arterial concentrations in the patients was compensated for by an increased fractional extraction for BCAA (Fig. 4), suggesting an increased blood-brain barrier permeability in the patients as found in previous studies with experimental animals (James et al. 1978). During administration of the BCAA mixture the brain uptake of leucine increased two- to threefold in both groups, but was significantly higher in the patients than in the healthy controls $(50 \%, P<0.05)$. The basal tyrosine and phenylalanine uptake seen in the patients was abolished 
and a similar tendency was noted also for methionine. The healthy controls displayed a similar pattern (Fig. 5).

\section{Clinical studies in patients with hepatic encephalopathy}

Experimental studies in man have thus verified essential parts of the plasma amino acid imbalance theory and positive effects of BCAA administration on central nervous system functions have been observed both in experimental studies (Smith et al. 1978; Higashi et al. 1981) and in uncontrolled clinical studies (Fischer et al. 1974, 1976). Despite this, the relation between the amino acid changes and hepatic encephalopathy remains doubtful. Most patients with liver cirrhosis have the typical changes in plasma amino acids and only minor differences are noted when patients without encephalopathy are compared with those with mild to moderate signs of encephalopathy (Fig. 6). A similar lack of relation between plasma amino acid changes and extent of encephalopathy has been reported both in cirrhotic patients (Morgan et al. 1978) and in patients with fulminant hepatic failure (Record et al. I976).

Results from adequately controlled clinical trials in patients with liver coma have so far been inconclusive. In a randomized, double-blind study, a BCAA solution was compared with glucose as placebo (Wahren et al. 1983). Significant decreases in plasma tyrosine, phenylalanine and methionine were observed in the BCAA-treated group. However, neither cerebral function nor survival was improved. Rossi-Fanelli et al. (1982) compared the efficacy of a BCAA-enriched solution and lactulose and concluded that the two forms of therapy were equally effective. Other studies, which have as yet only been reported in abstract form,

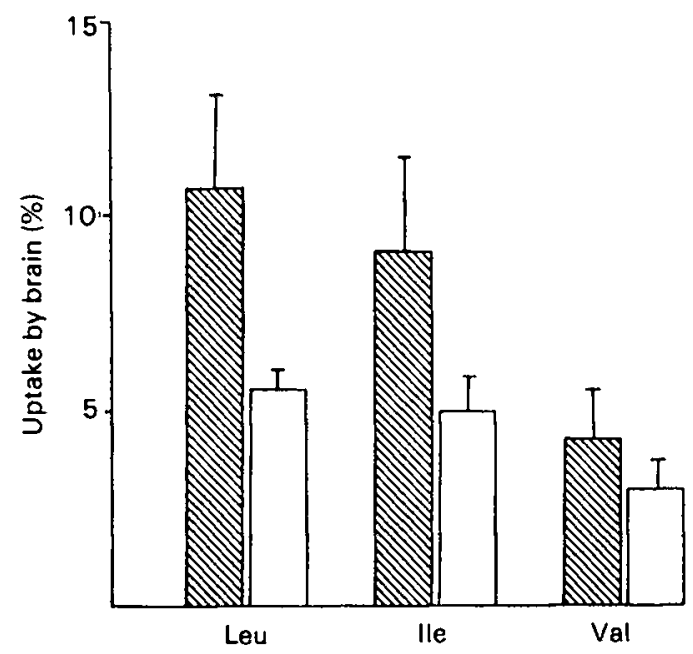

Fig. 4. Uptake of branched-chain amino acids across the brain in patients with liver cirrhosis (\$) and healthy controls $(\square)$. Standard errors of the means are represented by vertical bars. Significance of difference between patients and controls: leucine, $P<0.01$; isoleucine, $P<0.05$; valine, not significant. 


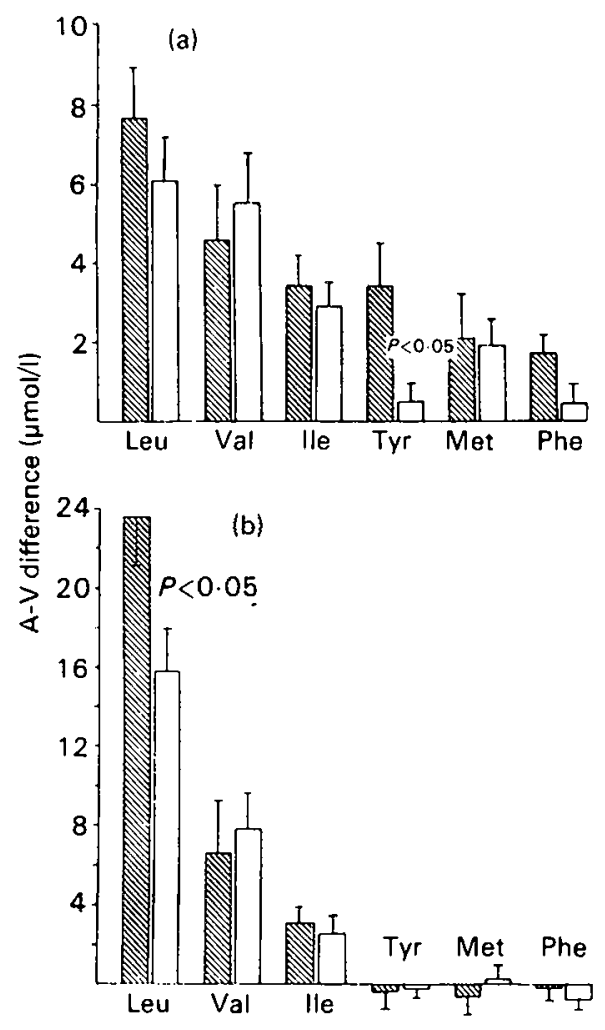

Fig. 5. Arterial-jugular venous $(\mathrm{A}-\mathrm{V})$ concentration differences of amino acids in patients with liver cirrhosis ( $($ ) and healthy controls $(\square)$ in (a) the basal state and (b) during branched-chain amino acid infusion. Standard errors of the means are represented by vertical bars.

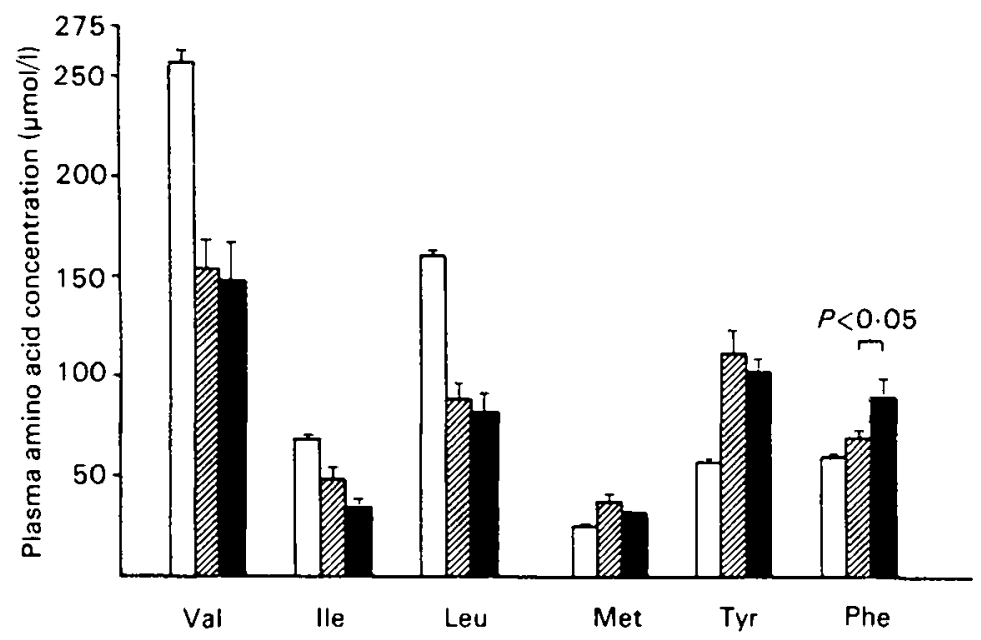

Fig. 6. Plasma amino acid levels in control subjects $(\square)$ and patients with liver cirrhosis without $($ G) and with mild to moderate ( $\square$ ) encephalopathy. Standard errors of the means are represented by vertical bars. 
have shown both negative (Michel et al. $\mathrm{I} 980$ ) and positive results (Cerra et al. I982).

Oral administration of BCAA has also been tried in patients with liver cirrhosis and chronic encephalopathy. In a double-blind, cross-over study, BCAA offered no advantage over placebo with regard to psychometric test or electroencephalogram results (Eriksson, Persson et al. 1982). This negative result was supported by a controlled study where ordinary protein was compared with a BCAA-enriched diet (Horst et al. i $98 \mathrm{I}$ ).

The plasma amino acid imbalance theory of portal-systemic encephalopathy has many attractive features and a vast amount of experimental evidence to support it has been presented. There remains only the last crucial step-to demonstrate that this new knowledge can be used to improve the treatment of patients with liver coma.

\section{REFERENCES}

Cerra, F. B., Cheung, N. K., Fischer, J. E., Kaplowitz, N., Schiff, E. R., Dienstag, J. L., Mabry, C. D., Leevy, C. M. \& Kiernan, T. (I982). Hepatology 2, 699.

Chatfield, C. \& Collins, A. J. (1980). Introduction to Multivariate Analysis. London: Chapman \& Hall.

Christensen, H. N. (1975). Biological Transport, and ed. Reading, Mass: W. A. Benjamin, Inc.

Collins, J. R. \& Crofford, O. B. (1969). Arch. Int. Med. 124, 142.

Eriksson, L. S., Hagenfeldt, L. \& Wahren, J. (1981). Clin. Sci. 60, 95.

Eriksson, L. S., Hagenfeldt, L. \& Wahren, J. (1982). Clin. Sci. 62, 285.

Eriksson, L. S., Persson, A. \& Wahren, J. (1982). Gut 23, 801 .

Eriksson, L. S., \& Wahren, J. (1982). Clin. Nutr. I, 127 .

Fischer, J. E. \& Baldessarini, R. J. (1971). Lancet ii, 75.

Fischer, J. E. \& Baldessarini, R. J. (1976). Progr. Liver Dis. 5, 363.

Fischer, J. E., Rosen, H. M., Ebeid, A. M., James, J. H., Keane, J. M. \& Soeters, P. B. (1976). Surgery $80,77$.

Fischer, J. E., Yoshimura, N., Aguine, A., James, J. H., Cummings, M. G., Abel, R. M. \& Deindoerfer, F. (1974). Am. F. Surg. 127, 40.

Guroff, G. \& Udenfriend, S. (I 622 ). F. biol. Chem. 237, 803.

Hagenfeldt, L. \& Arvidsson, A. (1980). Clin. Chim. Acta 100, 133.

Hagenfeldt, L., Eriksson, L. S. \& Wahren, J. (1980). Clin. Sci. 59, 173.

Higashi, T., Watanabe, A., Hayashi, S., Obata, T., Takei, N. \& Nagashima, H. (1981). In Metabolism and Clinical Implications of Branched Chain Amino Acids and Ketoacids. Developments in Biochemistry, vol. I8, p. 465 [M. Walser and J. R. Williamson, editors]. Amsterdam: Elsevier/North Holland.

Horst, D., Grace, N., Conn, H. O., Schiff, E., Schenker, S., Viberi, A., Law, D. \& Atterborough, C. E. (1981). Hepatology 1, 518.

Iber, F. L., Rosen, H., Levenson, S. M. \& Chalmers, T. C. (1957). F. Lab. Clin. Med. 50, $4^{1} 7$.

Iwasaki, Y., Sato, H., Ohkubo, A., Sanjo, T., Futagawa, S., Sugiura, M. \& Tsuji, S. (1980). Gastroenterology $78,677$.

James, J. H., Escourrou, J. \& Fischer, J. E. (1978). Science 200, I 395.

James, J. H., Ziparo, V., Jeppsson, B. \& Fischer, J. E. (1979). Lancet ii, 772.

Johnston, D. G. \& Alberti, K. G. M. M. (1977). Lancet i, to.

Krnjevic, K. (1974). Physiol. Rev. 54, 418.

Marchesini, G., Forlani, G., Zoli, M., Angiolini, A., Scolari, M. P., Bianchi, F. B. \& Pisi, E. (1979). Digest. Dis. Sci. 24, 594.

Michel, H., Pomier-Layrargues, G., Duhamel, O., Larombe, B., Guilleret, G. \& Bellet, H. (1980). Gastroenterology 79, 1038 . 
Miller, L. L. (1962). In Amino Acid Pools, p. 708 [J. T. Holden, editor]. Amsterdam: Elsevier/North Holland.

Morgan, M. Y., Marshall, A. W., Milsom, J. P. \& Sherlock, S. (1982). Gut $233^{62}$.

Morgan, M. Y., Milsom, J. P. \& Sherlock, S. (1978). Gut 18, 1068.

Munro, H. N., Fernstrom, J. D. \& Wurtman, R. J. (1975). Lancet i, 722.

O’Keefe, S. J. D., Abraham, R. R., Davies, M. \& Williams, R. (198r). Acta Chir. Scand. 507 (suppl.), 91 .

Oldendorf, W. H. \& Szabo, J. (1976). Am. F. Physiol. 230, 94.

Pardridge, W. M. \& Oldendorf, W. H. (1977). F. Neurochem. 28, 5.

Record, C. O., Buxton, B., Chase, R. A., Corzon, G., Murray-Lyon, I. M. \& Williams, R. (1976). Europ. J. clin. Invest. 6, 387 ,

Rossi-Fanelli, F., Riggio, O., Cangiano, C., Cascino, A., de Consiliis, D., Merli, M., Stortoni, M., Giunchi, G. \& Capoccia, L. (1982). Digest. Dis. Sci. 27, 929.

Rudman, D., DiFulco, T. J., Galambos, J. T., Smith, R. B., Salam, A. A. \& Warren, W. D. (1973). 7. clin. Invest. 52, 2241 .

Sato, Y., Eriksson, L. S., Hagenfeldt, L. \& Wahren, J. (I981). Clin. Physiol. I, I51.

Smith, A. R., Rossi-Fanelli, F., Ziparo, V., James, J. H., Perelle, B. A. \& Fischer, J. E. (1978). Ann. Surg. 187,343 .

Smith-Laing, G., Sherlock, S. \& Faber, O. (1 979). Gastroenterology 76, 685 .

Soeters, P. B. \& Fischer, J. E. (1976). Lancet ii, 880.

Wahren, J., Denis, J., Desurmont, P., Eriksson, L. S., Escoffier, J.-M., Gauthier, A. P., Hagenfeldt, L., Michel, H., Opolon, P., Paris, J. C. \& Veyrac, M. (1983). Hepatology (In the Press.)

Wu, C., Bollman, J. L. \& Butt, H. R. (x975). f. clin. Invest. 34, 845 .

Zinneman, H. H., Seal, U. S. \& Doe, R. P. (1969). Am. J. Digest. Dis. 14, 118. 